\title{
CINCO BIOGRAFÍAS ACADÉMICAS \\ EN EL 70 ANIVERSARIO DE LA REVISTA \\ ESTUDIOS DE EDAD MEDIA DE LA CORONA DE ARAGÓN
}

\begin{abstract}
FIVE ACADEMIC BIOGRAPHIES ON THE $70^{\text {th }}$ ANNIVERSARY OF THE JOURNAL ESTUDIOS DE EDAD MEDIA DE LA CORONA DE ARAGÓN
\end{abstract}

En este volumen de Aragón en la Edad Media hemos querido abrir tres secciones de contenidos - editorial, dossier y artículos - para celebrar el 70 aniversario de nuestra antigua revista, Estudios de Edad Media de la Corona de Aragón (EEMCA), fundada en 1945 por el profesor José María Lacarra. Su último número de una serie de diez se publicó en 1975. Dos años después, en 1977, la presente revista la sustituyó como publicación oficial del Departamento de Historia Medieval, Ciencias y Técnicas Historiográficas y Estudios Árabes e Islámicos de la Universidad de Zaragoza. Si EEMCA hubiera sido una profesora universitaria nacida en 1945 ahora en 2015 viviría su jubilación académica a los setenta años de edad. El símil no es baladí. Su cumpleaños coincide con el de una generación entera de personas que han dedicado la vida a la docencia, la investigación y la gestión en muchos centros e instituciones de educación superior. De hecho, en nuestro Departamento son cuatro profesores y una profesora todavía activos los que podemos considerar, si se me permite, de la «generación EEMCA». Aprovecharemos el 70 aniversario de la revista para rendirles homenaje con un dossier especial compuesto por sus cinco biografías académicas. Diversos autores y autoras los han retratado en una especie de exposición colectiva que muestra un quinteto de vidas paralelas que ahora se jubilan por imperativo de las fechas en que nacieron: Federico Corriente (14 de noviembre de 1940), Sebastián Andrés Valero (29 de noviembre de 1945), Asunción Blasco Martínez (10 de mayo de 1946), José Ángel Sesma Muñoz (7 de julio de 1946) y Esteban Sarasa Sánchez (14 de noviembre de 1946). 
De la trayectoria del Dr. Federico Corriente, catedrático emérito de Estudios Árabes e Islámicos y colaborador extraordinario del Departamento, se ocupan las profesoras María José Cervera y Ángeles Vicente, compañeras en su área de conocimiento. La historia académica del Dr. José Ángel Sesma, catedrático emérito de Historia Medieval, ha estado a cargo de su discípulo Mario Lafuente, profesor ayudante doctor. El retrato de la Dra. Asunción Blasco, catedrática de Ciencias y Técnicas Historiográficas, lo ha realizado Juan Antonio Barrio, profesor titular de Historia Medieval de la Universitat d'Alacant, no sólo por ser ella también alicantina sino especialmente por su legado de estudios sobre judíos y conversos de gran interés para el propio autor de la semblanza. En lo que concierne a la trayectoria del Dr. Esteban Sarasa, profesor titular de Historia Medieval, la persona elegida para redactarla ha sido la Dra. Ana Isabel Lapeña, que fue profesora de esta misma área de conocimiento en la Universidad de Zaragoza. Por último, el artículo sobre el Dr. Sebastián Andrés, profesor emérito de Historia Medieval, lo ha escrito el doctorando Raúl Villagrasa, máster en Investigación y Estudios Avanzados en Historia por la Universidad de Zaragoza.

El dossier no termina con esas cinco biografías académicas. El colofón del mismo lo pone un texto inédito con los índices de los diez volúmenes de la revista Estudios de Edad Media de la Corona de Aragón que recoge al final un listado alfabético de todos los autores que publicaron en la misma a lo largo de 1945-1975. Estos índices son un instrumento útil para conocer con detalle los contenidos de una revista que todavía no está digitalizada ni en acceso abierto al público, con lo que las dificultades de consulta son mayores. Esperamos que en un futuro próximo sea posible preparar la edición digital para su libre consulta en internet con las debidas autorizaciones legales. Estos índices de EEMCA que presentamos en el último texto del dossier van precedidos necesariamente por la advertencia escrita por el profesor José María Lacarra al comienzo del primer volumen de 1945 . No podía ser de otra manera. Ahí defendía la idea de fomentar y unificar los estudios que de manera dispersa se habían ido editando en multitud de publicaciones sobre la historia de Aragón en la Edad Media. El Consejo Superior de Investigaciones Científicas (CSIC) - que detenta la titularidad legal del EEMCA - había acordado crear el 27 de enero de 1943 el Centro de Estudios Medievales de Aragón (CEMA), incorporado después a la Escuela de Estudios Medievales del propio CSIC. La revista constituyó pues el órgano de expresión de un centro investigador ubicado en la Facultad de Filosofía y Letras de la Universidad de Zaragoza, siendo 
además el origen de una potente escuela de medievalistas bajo la batuta de Lacarra.

Para quienes deseen saber más sobre el Centro de Estudios Medievales de Aragón y la llamada Escuela de Medievalismo de Zaragoza pueden leer los artículos de María Isabel Falcón y José Ángel Sesma publicados en Medievalismo. Boletín de la Sociedad Española de Estudios Medievales, núms. 10 (2000) y 16 (2006), págs. 337-347 y 257-267 respectivamente. En esta editorial nos quedaremos con varias ideas importantes que provienen de ellos. El profesor Lacarra y el CEMA entendían ya entonces el concepto de Edad Media aragonesa no como algo local y restringido, sino abarcando toda la historia política e institucional de la Corona de Aragón y Navarra en la Edad Media en su sentido más amplio posible. Por ese motivo, la revista EEMCA no sólo recogió trabajos sobre el reino de Aragón, sino también de todos los países de la Corona e incluso del reino de Navarra, cuya historia en muchos momentos fue inseparable de la de Aragón, y así quedó latente en la advertencia del primer volumen de 1945. Por añadidura, las publicaciones derivadas de las tesis de licenciatura y doctorado de aquellos años engrosaron el contenido de la revista, concebida como portavoz de un espacio de investigación de elite que se integraba dentro del propio CSIC, a la vez que en el marco docente de la Facultad. La sección de la revista que aparecía al final de cada volumen bajo el epígrafe Información atrajo la atención de los principales centros de investigación medieval de ámbito internacional con los que se establecieron buenos contactos. Se comenzó también a reunir una biblioteca especializada en la que se incluyeron ejemplares de otras revistas con las que se mantuvieron intercambios.

Algún día habrá una tesis doctoral que escriba la historia del CEMA y del EEMCA y, por qué no, la de la revista actual Aragón en la Edad Media. Pero, por ahora y en espera de que así sea, nos conformaremos con visibilizar aquí una publicación cuyo legado intelectual ha llegado con fuerza hasta el presente. Una parte del profesorado del Departamento ha constituido en homenaje a aquel antiguo centro fundado por Lacarra un Grupo de Investigación Consolidado que se denomina precisamente CEMA, Centro de Estudios Medievales de Aragón, dirigido por José Ángel Sesma. De igual modo, el Área de Estudios Árabes e Islámicos ha creado otro Grupo Consolidado de Investigación denominado Árabe e Islam en Aragón que dirige Ángeles Vicente. Y también otra parte del profesorado ha constituido el Grupo Consolidado de Investigación Aplicada DAMMA, cuyas siglas corresponden a Documentos y Archivos 
Medievales y Modernos de Aragón, dirigido por Asunción Blasco. El legado investigador y gestor muy presente en el EEMCA está bien latente en el siglo XXI como puede verse, al margen incluso de otras iniciativas individuales o colectivas que no cito aquí pero que suman más esfuerzos, si cabe, para mostrar el dinamismo de nuestro Departamento. De igual modo, la presente revista, nuestra portavoz actual, convertida ya en una publicación digital en abierto dentro de la plataforma Open Journal System y con un consejo editorial de ámbito internacional, se sigue realizando paralelamente en versión impresa tan sólo por mantener un centenar de intercambios con otras revistas y publicaciones periódicas que nutren ahora la hemeroteca de la Biblioteca de Humanidades María Moliner de la Facultad de Filosofía y Letras. Antes era la biblioteca especializada de la Escuela de Estudios Medievales del CSIC, ubicada en el propio Departamento, la que tenía su propia hemeroteca y se alimentaba de los intercambios de entonces. Ahora, sin embargo, todo está centralizado y revierte a los fondos generales de la Biblioteca de la Facultad como sucede en todas partes. Pero el espíritu de la red permanente de intercambios de publicaciones sigue y seguirá en la medida de lo posible. Ése es un legado más del EEMCA.

Es interesante observar cómo el profesor Lacarra escribía en el año 1977 para la presentación del número 1 de nuestra revista Aragón en la Edad Media que los historiadores aragoneses hasta entonces habían sido muy parcos en adentrarse en la historia económica y social, más atentos a la historia de las instituciones claves del reino como la monarquía y las cortes. Tal vez con esas afirmaciones reconocía implícitamente que Estudios de Edad Media de la Corona de Aragón había potenciado sobre todo el análisis de aspectos políticos e institucionales al son de otros tiempos historiográficos. De hecho, para dejar bien claro el cambio de ruta la nueva revista se apellidó Estudios de Economía y Sociedad en los siete primeros volúmenes de 1977-1991. Incluso, la historia general de la Corona de Aragón y Navarra que había inspirado los volúmenes del EEMCA dejó paso a un interés preferente por el reino de Aragón a tono con aquellos años de efervescencia de la historia de las nuevas comunidades autónomas como la aragonesa, cuyo estatuto se aprobó en 1982. Eran los tiempos de las primeras historias, enciclopedias, compendios y atlas de Aragón como sucedía en otras partes del estado que comenzaban a hacer memoria colectiva de un rico pasado menospreciado por la visión centralista de la dictadura de Franco. En ese sentido, el EEMCA fue un motor de rehabilitación firme para la historia de la Corona de Aragón y Navarra a contracorriente del 
mundo académico coetáneo que le tocó vivir. Tan lejos llegó este otro legado suyo que finalmente se ha conseguido que Aragón en la Edad Media vuelva a defender aquel concepto amplio e integrador que en su día promovió el profesor Lacarra para sus Estudios, el de extender el ámbito de investigación de los artículos que puedan presentarse a todos los países de esa monarquía multiestatal, internacional y euromediterránea que fue la Corona de Aragón.

De todas estas cosas que he dicho hablarían con mayor conocimiento de causa por su excelente bagaje personal y profesional nuestros queridos colegas y amigos Sebastián Andrés, Asunción Blasco, Federico Corriente, Esteban Sarasa y José Ángel Sesma. Ellos y ella han sido testimonios directos con sus vidas en paralelo de lo que fue investigar en aquellos años sesenta y setenta que vieron el final de un tiempo político y de una manera oficial de entender la historia bastante diferente a lo que ha venido después. El desarrollo de sus carreras académicas en los años ochenta, noventa y en los inicios del siglo XXI les habrá producido cierta sensación de vértigo, de demasiados cambios respecto al mundo de sus maestros, que es el suyo propio. Pero aquí y ahora siguen activos con una mirada privilegiada sobre todo lo acontecido. $\mathrm{Y}$ al igual que ellos se parecen más a sus maestros que a sus discípulos, sus discípulos se parecen más a ellos que a los que vengan después. Algo así es un símbolo claro de continuidad, que no de continuismo.

Al día de hoy, uno de los valores más grandes que atesoran nuestros homenajeados es sin duda el de la mirada en profunda retrospectiva sobre unos tiempos que ya no volverán. Es como estar ya en lo alto del monte y divisar todas las tierras alrededor con la seguridad que permite la sabiduría y el equilibrio. A pesar de ello, es probable que cuando imaginen el futuro crean que lo que pueda suceder ya no tenga mucho que ver con lo que ellos vivieron, pero habrá algo que sí seguirá conectando su quehacer y sus vidas con las de generaciones futuras. Me refiero a los valores del esfuerzo, la responsabilidad, la cooperación, el intercambio positivo de saberes y, especialmente, el respeto a las generaciones precedentes en lucha abierta contra la gerontofobia. Todo ello deberá seguir existiendo porque sin esa ideología académica, sin ese vaivén entre la ciencia y la vida, la universidad no será universidad. En suma, que les queda mucho por delante, que necesitamos su presencia para seguir viviéndolos como referentes a imitar. Sucede algo parecido con nuestra revista de hoy, que necesita recuperar de vez en cuando el espíritu académico que forjó aquella magna serie de Estudios de la Edad Media de la Corona de Aragón, pionera en interdisciplinariedad e internacionalización. Vaya pues todo el ca- 
riño, el afecto y el reconocimiento del Consejo Editorial de nuestra revista y de todo el Departamento hacia quienes en estos años van a ver en uno u otro instante su jubilación académica, que será a todas luces una nueva etapa llena de retos y plena de vida.

Germán Navarro Espinach

Director de la Revista Aragón en la Edad Media 